\title{
The treatment of post-traumatic facial skin defect with artificial dermis
}

\author{
Ki-Sung Park, \\ Wu-Seop Lee, \\ So-Young Ji, \\ Wan-Suk Yang \\ Department of Plastic and Reconstructive \\ Surgery, Dong Kang General Hospital, Ulsan, \\ Korea
}

\begin{abstract}
Background: When a skin defect occurs, clinicians must work to restore the original skin quality as soon as possible. Accordingly, an artificial dermis can be used to supplement the wound and prevent severe scar contracture formation. The Terudermis is an artificial dermis that is simple and easy to use. We investigated the effectiveness of the Terudermis in the treatment of facial skin defects by analyzing previous relevant cases treated in our institution.

Methods: We retrospectively examined 143 patients who were treated with the Terudermis graft in facial skin defect at Dong Kang General Hospital in 2015 and 2016. The patients' age, sex and location, wound size, complications were analyzed. In addition, the patients were asked to complete a self-satisfaction questionnaire after 18 months from the completion of treatment. The results were compared with that of autologous full-thickness skin graft (FTSG) and split-thickness skin graft (STSG) patients in same period.

Results: The mean self-satisfaction scores evaluated by patients were $4.1 \pm 1.0,4.0 \pm 1.3$ and $3.5 \pm 1.8$ for the Terudermis graft, FTSG and STSG patients, respectively. With respect to complications, there were fewer incidences of hematoma, partial skin loss and complete skin loss in the Terudermis graft patients.

Conclusion: In the present study, the Terudermis, when used to treat post-traumatic facial skin defects, is a good alternative option to obtain satisfactory aesthetic outcomes. Also, the Terudermis grafting is a simple and easy treatment method to perform.
\end{abstract}

Keywords: Artificial dermis / Skin defect / Terudermis

\section{INTRODUCTION}

The skin acts as a barrier that protects the body from the external environment and prevents water loss [1]. When a skin defect occurs, clinicians must use treatments that promote quick healing and rapidly restore the original structure of the skin. Indeed, if a wound remains open long-term, it may be vulnerable to secondary complications such as infection, and the patient may experience pain and discomfort throughout the treatment period. Moreover, the development of a prominent scar can lead to severe functional and cosmetic problems, even after treatment has ended. The

\section{Correspondence: Wan-Suk Yang}

Department of Plastic and Reconstructive Surgery, Dong Kang General Hospital,

239 Taehwa-ro, Jung-gu, Ulsan 44455, Korea

E-mail: artps@naver.com

Received October 11, 2017 / Revised March 14, 2018 / Accepted March 15, 2018 wound healing process proceeds gradually and eventually restores the skin barrier. This process is complex and involves a variety of cells. During wound healing, the dermis of the damaged skin is replaced by scar tissue, which epithelializes to form wound borders. In this regard, the amount and arrangement of the dermal elastic fibers have great effect on the quality of the restored skin [2,3]. For instance, split thickness skin graft (STSG) yields patchy remnants of the dermal papillae, which do not provide a healthy dermal layer for the wound. Therefore, STSG may lead to unsatisfactory outcomes such as scar contraction [4]. Skin defects caused by a crushing injury, such as those that occur in traffic accidents or falls, have even worse viability. For this reason, such injuries are associated with a high risk of skin loss if an STSG or full thickness skin graft (FTSG) is performed immediately. To treat skin defects, surgeons often graft using artificial dermis first to reduce the risk of later 
skin loss and somewhat prevent the severe scar contraction that can occur after skin reconstruction. The artificial dermis consists of collagen, proteoglycans, and elastic fibers. It limits scar formation and promotes dermal tissue formation, thereby preventing scar contracture formation $[5,6]$. The artificial dermis has a highly permeable structure with many pores, and its regenerative ability is determined by factors such as chemical composition, pore size and porosity, and degradation rate. One of the available artificial dermis products, the Terudermis (Terumo, Tokyo, Japan), can be used to replace lost dermis in a simple procedure that is performed before autologous skin grafting, providing volume and forming a bed to which the later skin graft can adhere.

Here we aimed to collect cases that showed epithelialization after the Terudermis was used to treat a post-traumatic facial skin defect and discuss the potential of this procedure based on these experiences.

\section{METHODS}

We retrospectively examined the charts that covered 143 patients who were treated with the Terudermis graft in their facial skin defect at Dong Kang General Hospital in 2015 and 2016. Only patients whose skin defects were caused by trauma were selected in this study. Clinical information such as the patients' age, sex and location, size, complications were researched. Since the Terudermis is a substitute for the dermal layer of skin, we generally have used it in patients with full-thickness skin defects. The surgical method was selected by the patients' preference with detailed information on the artificial dermis graft procedure, including surgical techniques, advantages, and disadvantages but not by initial wound status. Eighteen months after wound healing, the patients were asked to complete a self-satisfaction questionnaire. Scores were defined as 1, poor; 2 , fair; 3 , good; 4, very good; and 5, excellent. Also, to compare clinical outcomes of the Terudermis graft, we evaluated 38 patients whose facial skin defects were treated using autologous FTSG and 21 patients whose facial skin defects were treated using immediate STSG in same period. This study adhered to the principles stated in the Declaration of Helsinki, and the protocol was approved by Institutional Review Board/
Ethics Committee of Dong Kang General Hospital.

\section{Operation methods}

After necrotic soft tissues and foreign bodies around the wound were carefully debrided, the Terudermis was cut using scissors to fit the defect (Fig. 1). It was then placed with the silicone membrane facing upward and secured to the defect using 6-0 nylon fixation. For the dressing, the Terudermis was first covered with saline-soaked gauze and then with a layer of dry gauze to maintain the moist environment. The initial dressing was applied on postoperative day 1 . The wound was checked for infection and the dressing was changed once a day. At postoperative day 14, after the Terudermis had been replaced by a pseudo-dermis, the silicone membrane was removed, and STSG was performed on the recipient site.

\section{Statistical analysis}

The statistical analysis was performed using IBM SPSS ver. 22.0

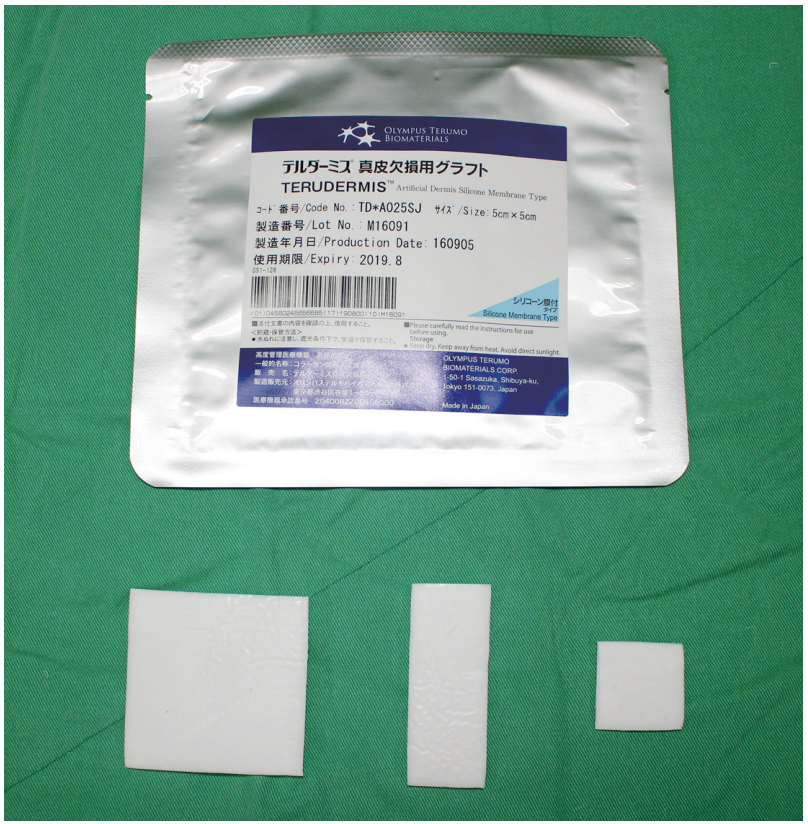

Fig. 1. The Terudermis can be cut into various shapes and sizes before it is affixed to the wound bed. 
(IBM Corp., Armonk, NY, USA). Pearson's chi-square test and the Student $t$-test were performed to evaluate the data in this study. The $p$-values $<0.05$ were considered statistically significant.

\section{RESULTS}

Among the 143 patients whose skin defects were treated with the Terudermis graft, there were 97 males and 46 females aged 8 to 91 years (Table 1). The mean wound size was $28.8 \times 34.5 \mathrm{~mm}^{2}$ for the Terudermis graft patients. The major causes of trauma were bicycle and motorcycle accidents (32\%), falls (24\%), and violence (11\%) (Fig. 2). Cheek, nose and forehead were major locations of facial skin defect treated by the Terudermis graft (Table 2). The mean self-satisfaction scores evaluated by patients were 4.1 $\pm 1.0,4.0 \pm 1.3$ and $3.5 \pm 1.8$ for the Terudermis graft, FTSG and STSG patients, respectively (Table 3). With respect to the incidence of complications, there were ten hematomas, eight partial skin losses and two complete skin losses in the Terudermis graft patients (Table 4).

Table 1. Patient characteristics

\begin{tabular}{|c|c|c|c|}
\hline Variable & $\begin{array}{l}\text { Terudermis } \\
(n=143)\end{array}$ & $\begin{array}{l}\text { FTSG } \\
(n=38)\end{array}$ & $\begin{array}{l}\text { STSG } \\
(n=21)\end{array}$ \\
\hline Age, range (yr) & $8-91$ & 9-79 & $15-75$ \\
\hline Sex (male: female) & $97: 46$ & $24: 14$ & $15: 6$ \\
\hline $\begin{array}{l}\text { Wound size }\left(\mathrm{mm}^{2}\right) \\
\text { Min } \\
\text { Max } \\
\text { Mean }\end{array}$ & $\begin{array}{c}5 \times 6 \\
48 \times 65 \\
28.8 \times 34.5\end{array}$ & $\begin{array}{c}7 \times 6 \\
21 \times 19 \\
17.8 \times 15.5\end{array}$ & $\begin{array}{c}12 \times 14 \\
45 \times 52 \\
30.1 \times 37.6\end{array}$ \\
\hline $\begin{array}{l}\text { Mean treatment } \\
\text { duration (day) }\end{array}$ & 19 & 13 & 17 \\
\hline
\end{tabular}

FTSG, full thickness skin graft; STSG, split thickness skin graft.

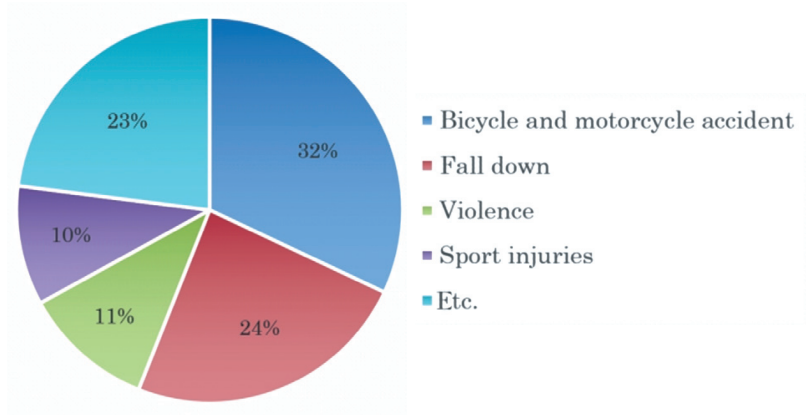

Fig. 2. The proportion of trauma incidents which caused facial skin defect.

\section{Case 1}

A 53-year-old man had a 21 $\times 17 \mathrm{~mm}^{2}$ full-thickness skin defect on the nasal dorsum after a bicycle accident (Fig. 3). After debridement and wound irrigation, the Terudermis was cut to the defect size and fixated immediately in the emergency room. The silicone membrane was removed after 14 days and STSG was performed using the epidermal layer of the forehead skin. The patient graded his scar outcome as 4.0 (good) and was cosmetically satisfied.

\section{Case 2}

A 15 -year-old boy had a $5 \times 6 \mathrm{~mm}^{2}$ of skin defect on the medial canthus after he was pricked by a tree branch (Fig. 4). To improve the appearance of the upper eyelid margin and fold, the Terudermis graft was performed immediately at the emergency room. The wound was well healed without STSG because the re-epithelization on the Terudermis was sufficient to cover the defect after postoperative day 14 . The patient graded his scar outcome as 4.2 (very good) and was cosmetically satisfied.

\section{DISCUSSION}

When a skin defect occurs, an FTSG or local flap is used for reconstruction. To cover a large-sized defect, the dermis can be reconstructed using an STSG, while an FTSG is ineffective [7]. However, in crushing injuries, which involve a poor condition of the wound bed with various infection sources like foreign bodies, immediate autologous skin grafts are at high risk of failure. Furthermore, if the volume loss is severe enough to expose bone and tendon, an STSG alone is unlikely to be successful and a depression can develop after recovery, resulting in poor esthetic outcomes. An artificial dermis can be used to overcome these drawbacks, and it yields satisfactory outcomes by promoting healthy vascularization prior to skin grafting.

Here we used the Terudermis, which possesses a dual structure similar to that of the Integra, the first artificial dermis developed by Yannas and Burke [8] in 1980. The Terudermis promotes dermal regeneration by acting as a template for capillary growth and 
Table 2. Location of facial skin defect treated by the Terudermis graft

\begin{tabular}{cccccccccc} 
Location & Cheek & Nose & Temple & eyelid & Lip & Preauricular & Forehead & Chin & Total \\
Patient & 33 & 26 & 19 & 11 & 9 & 6 & 24 & 15 & 143 \\
\hline
\end{tabular}

Table 3. Comparison of patient's self-satisfaction

\begin{tabular}{lccc} 
Variable & Terudermis $(n=143)$ & FTSG $(n=38)$ & STSG $(n=21)$ \\
Self-satisfaction & $4.1 \pm 1.0$ & $4.0 \pm 1.3$ & $3.5 \pm 1.8$ \\
\hline
\end{tabular}

Values are presented as mean \pm standard deviation.

FTSG, full thickness skin graft; STSG, split thickness skin graft.

Table 4. Comparison of complication incidence

\begin{tabular}{lccc} 
Complication & Terudermis $(\mathrm{n}=143)$ & FTSG $(\mathrm{n}=38)$ & STSG $(\mathrm{n}=21)$ \\
Hematoma & $10(6.9)$ & $5(13.1)$ & $5(23.8)$ \\
Partial skin loss & $8(5.5)$ & $2(5.2)$ & $3(14.2)$ \\
Complete skin loss & $2(1.3)$ & $1(2.6)$ & $1(4.7)$ \\
Total & $20(13.9)$ & $8(21.0)$ & $9(42.8)$ \\
\hline
\end{tabular}

Values are presented as number (\%).

FTSG, full thickness skin graft; STSG, split thickness skin graft.
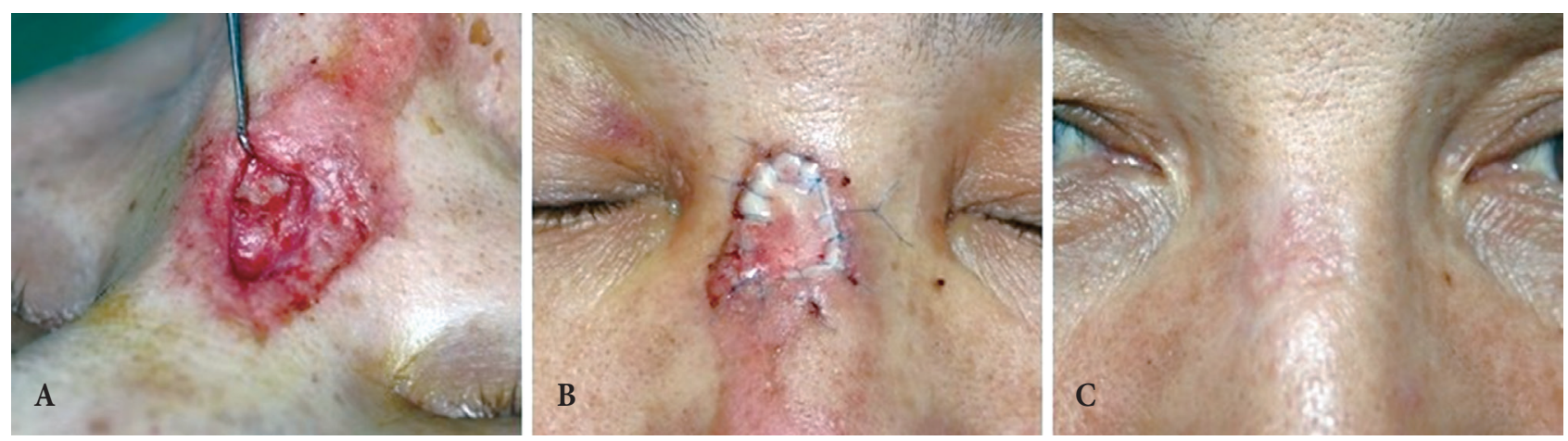

Fig. 3. Case 1. (A) A 53-year-old man had a $21 \times 17 \mathrm{~mm}^{2}$ of full-thickness skin defect on the nasal dorsum after a bicycle accident. (B) Postoperative view at 1 day after the Terudermis grafting. (C) Postoperative view at 18 months of follow-up.
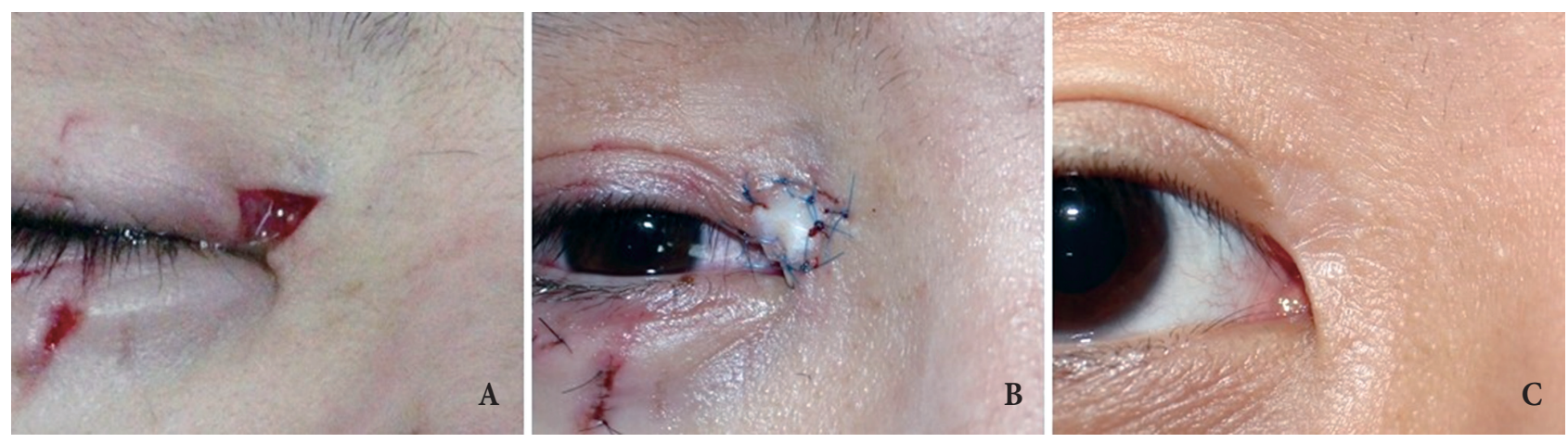

Fig. 4. Case 2. (A) A 15-year-old boy had a $5 \times 6 \mathrm{~mm}^{2}$ of skin defect on the medial canthus after he was pricked by a tree branch. (B) Postoperative view at 1 day after the Terudermis grafting. (C) Postoperative view at 19 months of follow-up. 
infiltration. It is a collagen sponge composed of heat-denatured fibrillar atelocollagen that has been dehydrothermally crosslinked [9]. In the present study, the artificial dermis was accepted soon after implantation, resulting in fewer contractures as well as longterm survival. This was because a collagen sponge with dehydrothermal crosslinks attracts fibroblastic cells during the early phase of the host-implant reaction and is eventually replaced by host tissue [10]. Ultimately, the implanted sponge gains a uniform stromal appearance changed by the host's fibroblasts. This can increase the thickness of the dermis and improve acceptability at the site of subcutaneous tissue defects or of cartilage or tendon exposure [11]. When a traumatic wound occurs on the face, surgeons can choose from among several treatment methods. This decision is based on patient age as well as wound size, color, and texture.

The usefulness of the Terudermis has been demonstrated through repeated clinical use since its development 20 years ago. It is typically used to replace the dermis during the intermediate stage of wound healing, and treatment is completed-after a certain time has passed-during the second stage, in which a layer of the patient's epidermis is grafted over the Terudermis. In the present study, we debrided the necrotic tissue, suture-fixed the Terudermis, and dressed the wound daily with careful observation. STSG is typically performed 2 weeks after Terudermis placement. However, in some cases, dressing treatment alone was sufficient to achieve wound healing without additional skin grafting. Using Terudermis grafting is a relatively simple and easy procedure. Because the Terudermis does not contain an epidermis layer, the STSG is usually performed after 2 weeks of dressing. Open wounds are vulnerable to dryness, infection, and contamination, so an autologous skin graft should be used to treat wounds above a certain size to enable rapid complication-free healing. However, we have observed cases in which small wounds showed rapid epithelialization, and additional treatment was unnecessary after 2-3 weeks of management.

When we analyzed cases treated using the Terudermis, the esthetic outcomes of scars after the completion of treatment were satisfactory. A patient's esthetic satisfaction could be further increased using laser therapy, which alleviates pigmentation. Relatively small facial skin defects can also be treated using local flap surgery after debridement and thorough undermining to reduce tension. However, undermining does not remove tension entirely and can lead to necrosis at the skin margin. Dog ears can also develop at either end of the wound after closure, and a linear elevation may develop along the scar. Moreover, in some deformities, especially those around the nose, the skin cannot be advanced because it lacks elasticity. Similarly, with lesions around the lips and eyes, cases in which greater care is required because scars are readily visible and keep moving, primary closure is often not an appropriate choice. Terudermis grafting can be used to avoid these difficulties.

Nonetheless, there are drawbacks to treatment using Terudermis grafting. For example, the dressing placed prior to skin grafting must be maintained for approximately 2 weeks, and repeat surgery is occasionally required because the Terudermis sometimes dissolves due to infection. In addition, wound depth varies among individual cases, so Terudermis thickness must be considered to minimize later scar depression. Several Terudermis layers can be used to fill deeper wounds. It then takes longer for the complex structure of neovascularization and autologous collagen to form. Further research is required to explore methods that promote Terudermis survival in such cases. In addition, we must admit that there are some biases regarding the subjects of comparison. Surgery is performed immediately or within 3 to 4 days of the injury when FTSG or STSG is used, while dressing treatment is first performed for about 2 weeks after Terudermis grafting before the STSG is repeated. During these 2 weeks, some granulation tissue regenerates and the wound healing process proceeds, which is an advantage of the Terudermis in terms of the incidence of wound complications. Despite subject objectivity being slightly undermined, we conducted this study because the Terudermis treatment could lead to substantially satisfactory outcomes in terms of scar quality and esthetic outcomes. In conclusion, to treat facial skin defects, it is well known that the best treatment choice is usually local flap, if possible, and FTSG or STSG can be used in relatively large skin defects depending on the donor site limitation. The Terudermis which can be applied to various wound sizes must be a good alternative option to treat such facial skin defects with enhanced esthetic outcome. Also, the Terudermis is 
worth mentioning for its low cost and convenience of use to treat post-traumatic skin defects.

\section{CONFLICT OF INTEREST}

No potential conflict of interest relevant to this article was reported.

\section{PATIENT CONSENT}

The patients provided written informed consent for the publication and the use of their images.

\section{REFERENCES}

1. Min JH, Yun IS, Lew DH, Roh TS, Lee WJ. The use of matriderm and autologous skin graft in the treatment of full thickness skin defects. Arch Plast Surg 2014;41:330-6.

2. Yannas IV, Burke JF, Gordon PL, Huang C, Rubenstein RH. Design of an artificial skin. II. Control of chemical composition. J Biomed Mater Res 1980;14:107-32.

3. Juhasz I, Kiss B, Lukacs L, Erdei I, Peter Z, Remenyik E. Long-term followup of dermal substitution with acellular dermal implant in burns and postburn scar corrections. Dermatol Res Pract 2010;2010:210150.

4. Cervelli V, Brinci L, Spallone D, Tati E, Palla L, Lucarini L, et al. The use of MatriDerm ${ }^{\circledR}$ and skin grafting in post-traumatic wounds. Int Wound J 2011;8:400-5.

5. Hatoko M, Kuwahara M, Tanaka A, Tada H, Yurugi S, Mashiba K, et al. Application of artificial dermis graft to correct a depression after tissue resection. Ann Plast Surg 2000;45:633-40.

6. Yim H, Cho YS, Seo CH, Lee BC, Ko JH, Kim D, et al. The use of AlloDerm on major burn patients: AlloDerm prevents post-burn joint contracture. Burns 2010;36:322-8.

7. Tufaro AP, Buck DW 2nd, Fischer AC. The use of artificial dermis in the reconstruction of oncologic surgical defects. Plast Reconstr Surg 2007;120:638-46.

8. Yannas IV, Burke JF. Design of an artificial skin: I. basic design principles. J Biomed Mater Res 1980;14:65-81.

9. Hatoko M, Tanaka A, Kuwahara M, Tada H, Muramatsu T. Use of artificial dermis graft for covering the femoral vessels after groin dissection. Eur J Plast Surg 1999;22:400-3.

10. Koide M, Osaki K, Konishi J, Oyamada K, Katakura T, Takahashi A, et al. A new type of biomaterial for artificial skin: dehydrothermally cross-linked composites of fibrillar and denatured collagens. J Biomed Mater Res 1993;27:79-87.

11. Yurugi S, Hatoko M, Kuwahara M, Tanaka A, Iioka H, Niitsuma K. Usefulness and limitations of artificial dermis implantation for posttraumatic deformity. Aesthetic Plast Surg 2002;26:360-4. 